\title{
IRON SUPPLEMENTATION IN TREATMENT OF CHILDREN WITH LOW RESPIRATORY INFECTION AND IRON DEFICIENCY ANEMIA
}

\author{
Gonchar M. ${ }^{1}$, Ischenko T. ${ }^{1}$, Tyshko A. ${ }^{1}$, Orlova ${ }^{1}{ }^{1}$, Saenko.$_{\bullet}{ }^{1}$, Bondar ${ }^{2}{ }^{2}$ \\ Kharkiv National Medical University, Ukraine ${ }^{1}$, \\ Kharkiv Regional Children's Clinical Hospital, Ukraine ${ }^{2}$
}

\begin{abstract}
Upper respiratory tract infections as well as bronchitis and pneumonia are most common causes for hospitalization of children. Younger children are more predisposed to these problems and its complications. Anemia is one of the most common comorbidity of this pathology. We analyzed 52 case histories of children diagnosed with pneumonia and deficiency anemia. The study evaluated presentation, history, clinical and laboratory data. Our results suggest that ferrotherapy helps with acceleration of low respiratory tract infections' symptoms
\end{abstract}

Key words: lower respiratory tract infection, anemia, children, iron supplement.

Introduction. Infection of the lower respiratory tract is the main cause of mortality in children worldwide due to developing complications, in the form of intoxication and obstructive syndromes. Pneumonia is the leading cause of death among children under 5 years [1]. The estimated incidence in this age group in developed countries is 1 in 15 episodes per child year [2]. This amounts to about 156 million new cases for the first time per year per child population [3]. One of the most common comorbidities of the low respiratory tract infections is deficient anemia, mainly iron deficiency anemia. 50\% of all cases of deficient anemia are iron deficiency states and in the opinion of many authors, iron deficiency anemia is one of the most common contributing factors in pneumonia [4].

Anemia is a group of clinical hematological syndromes, the general criterion of which is a decrease in the concentration of hemoglobin and/ or erythrocytes per unit volume of blood [4].

According to the literature [5], the pathogenic mechanism for the development of microbial inflammatory diseases in patients with anemia is associated with oxygen starvation of tissues, and with trophic disorders in the mucosal endothelium walls, leading to a decrease in the protective functions of the endothelium, including the secretory immunoglobulin A.

In addition, the effects of iron deficiency also lead to [6]:

Corresponding Author:

Tatiana Ishenko, MD, PhD, Associate Professor, Department of Pediatrics 1 and Neonatology, Kharkiv National Medical University, Ukraine. E-mail: 7tanyatb7@gmail.com
- violation of neuro-psychological functions in children;

- decrease in intellectual development indices;

- retardation of the development of logical thinking and speech with potential learning difficulties;

- degenerative-dystrophic changes in the epithelium of the skin, mucous membranes of the gastrointestinal tract, respiratory tract, which is one of the reasons for the development and progression of microbial inflammatory diseases.

Since the role of anemia in the development of microbial inflammatory diseases of the lower respiratory tract is undeniable, it is worth knowing the causes and early signs of anemia.

The causes of anemia in young children include: multiple pregnancies, iron deficiency anemia in the mother during pregnancy, bleeding during pregnancy and childbirth in the mother and/ or child, the use of unadapted mixtures, cow or goat milk for feeding, qualitative and quantitative non-correlation of feed. according to the age.

The clinical signs of anemia include: sideropenic and anemic syndromes. Thus, early signs of anemia: lethargy, emotional weakness, psychomotor retardation, increased heart rate, weakening of heart tones, functional systolic murmur, tachypnea, prolonged low-grade fever, loss of appetite or lack of it, dry skin, dullness, hair loss, fragility, layering of nails, stomatitis, perversion of taste and smell.

2. Purposes, subjects and methods:

2.1. Purpose - to establish the relationship of iron deficiency anemia and infection of the lower respiratory tract in young children. 
2.2. Subjects \& Methods. A retrospective study was conducted over two years (2015-2017) for children aged from 3 months to 35 months who were admitted to Kharkiv Regional Children Clinical Hospital. The study included 82 diagnosed cases of infections of the lower respiratory tract: 52 patients diagnosed with acute pneumonia and iron deficiency anemia (group I) and 30 patients diagnosed with acute pneumonia without anemia (group II).

Patients with prematurity, chronic inflammatory diseases, dietary disorders and severe systemic diseases, despite the presence of diagnostic criteria for pneumonia and iron deficiency anemia were excluded from the study.

Standard diagnostic protocol was applied, which included assessment of history and clinical data, chest radiography and sputum culture. CBC with platelet count, reticulocyte count and color index allowed to establish the etiology of anemia as well as iron level in the blood.

The following equation [7], which is especially relevant when evaluating $\mathrm{CBC}$ performed by using a hardware hemo-analyzer, was used to calculate the color index:

color index $=3 *(\mathrm{Hb}$ in $\mathrm{g} / \mathrm{l}) /$ three first digits of the number of erythrocytes

Conflict of interests. There is no conflict of interests.

3. Results and discussion. Patients were equally distributed by age $(2.9 \pm 0.4 \mathrm{vs} .2 .9+0.6$ years, $\mathrm{p}<0.05)$ and $\operatorname{sex}(60.4$ vs. $57.9 \%$ of boys, $\mathrm{p}<0.05$ ).

In both groups, respiratory syndrome, broncho-obstructive syndrome and intoxication were recorded in patients with the same frequency. Pneumonia was confirmed radiologically in $100 \%$ patients of both groups. There were no differences in the degree of inflammation by CBC data: an increase in the number of neutrophils $(12.4 \pm 3.3$ vs. $11.7 \pm 4.1 \times$ $\left.\times 10^{9}, \mathrm{p}<0.05\right)$, and accelerated ESR $(22.3 \pm 1.3$ vs. $19.3 \pm 0.9 \mathrm{~mm} /$ hour, $\mathrm{p}<0.05$ ).

In group I clinical and laboratory data corresponded to moderate deficiency anemia: hemoglobin ( $83 \pm 0.2 \mathrm{~g} / \mathrm{l}$ ), hypochromic RBC (color index $0.64 \pm 0.03 \mathrm{cu}$ ) with normoregenerative state (level of reticulocytes $1.2 \pm 0.1 \%$ ).

Interestingly, that decreased iron level (up to $6.4 \pm 0.3$ ? mol/l) with no CBC changes was detected in Group II patients, which allowed us to diagnose latent sideropenia.

In therapy, patients of both groups received symptomatic and antibacterial therapy, according to the relevant recognized protocols. In addition, patients of group I received iron supplement therapy with $\mathrm{Fe} 2$ + at a dose of $5 \mathrm{mg} / \mathrm{kg} / 24 \mathrm{~h}$.

As a result of the treatment, Group I patients were found to have a reduction of clinical symptoms of bronchial obstruction in $2.2 \pm 0.2$ days, and intoxication syndrome in $2.1 \pm 0.4$, earlier than Group II patients ( $<<0.05$ for both).

\section{Conclusions:}

1. Lower respiratory infections are associated with the presence of anemia and sideropenic states in young children. That is why it is advisable to perform detailed CBC test (with platelets, reticulocyte count and color index) and to determine the iron level if indicated for the early diagnosis of iron deficiency states in young children with low respiratory tract infections.

2. Early diagnosis of iron deficiency and prevention of anemia are important measures for reducing the incidence of lower respiratory infections in young children.

3. Iron supplement therapy helps to reduce the symptoms of low respiratory tract infections.

\section{References:}

1. Moore, Michael et al.(2017) "Predictors of pneumonia in lower respiratory tract infections: 3C prospective cough complication cohort study" European respiratory journal vol. 50,5 1700434. 23 Nov. 2017, doi:10.1183/13993003.00434-2017

2. Rudan, I., Boschi-Pinto, C., Biloglav, Z., Mulholland, K., \& Campbell, H. (2008). Epidemiology and etiology of childhood pneumonia. Bulletin of the World Health Organization, 86(5), 408-16.

3. Rudan, Igor et al.(2008) "Epidemiology and etiology of childhood pneumonia" Bulletin of the World Health Organization vol. 86,5 (2008): 408-16.

4. Nepalese Medical Journal, (2018) Vol. 1, 5-8 Anemia as a Risk Factor for Acute Lower Respiratory Tract Infection inChildren Below Five Years of Age.Shakya Henish,1 Singh Saurav,1 Lakhey Ashish,)

5. Sawsan Mourad, MD., Mariam Rajab, MD., Aouni Alameddine, MD., Mohammad Fares, MD., Fouad Ziade, PhD., Bassem Abou Merhi, MD.(2010). Hemoglobin level as a risk factor for lower respiratory tract infections in Lebanese children North American Journal of Medical Sciences N Am J Med Sci. 2(10): 461-466.

6. Gupta, Priya M et al.(2026) "Iron, Anemia, and Iron Deficiency Anemia among Young Children in the United States" Nutrients vol. 8,6 330. 30 May. 2016, doi:10.3390/nu8060330

7. Joo, Eun Young et al. (2016)"Iron deficiency anemia in infants and toddlers" Blood research vol. 51,4 (2016): 268-273.

Received: 15-Oct. - 2018

Accepted: 09-Dec. - 2018 